\title{
3D reconstruction of ultrasound scanned data for tissue mimicking material sample
}

\author{
Haijiang Zhu ${ }^{\mathrm{a}, *}$, Ping Yang ${ }^{\mathrm{b}}$ and Tianbin $\mathrm{Yao}^{\mathrm{a}}$ \\ ${ }^{a}$ College of Information Science \& Technology, Beijing University of Chemical Technology, Beijing \\ 100029, China \\ ${ }^{b}$ Division of Mechanics and Acoustic, National Institute of Metrology, Beijing 100013, China
}

\begin{abstract}
This paper investigates a 3D reconstruction based on the ultrasonic scanned data for tissue mimicking material (TMM) sample. A two-step varied window filter is developed to smooth ultrasound backscatter signals at first. Next, the anisotropic diffusion filter with a triangular window is presented to reduce the noise of the 2D images by aligning onedimensional signals. Finally, the 3D structure of the object embedded in the TMM sample is reconstructed using the detected edges images. The performance of the proposed method is analyzed and validated through a number of experiments in both $2 \mathrm{D}$ imaging and $3 \mathrm{D}$ reconstruction.
\end{abstract}

Keywords: 3D ultrasound reconstruction, tissue mimicking materials, two-step varied window filter, anisotropic diffusion filter

\section{Introduction}

The prostate, liver and muscle tissue mimicking materials (TMM) have been widely used in the field of assessing the performance of ultrasound scanners, ultrasound training simulator and ultrasound-guided puncture techniques in recent years. However, 2D ultrasound images in these fields only show a cross-section of a lesion and cannot describe its spatial location. Therefore, many commercial applications and researchers have paid close attention to 3D ultrasound imaging.

3D ultrasound imaging has attracted more and more researchers to observe spatial structures and details of an examined object in the past ten years [1]. There exist three kinds of technologies for reconstructing 3D ultrasound images in the reported literature according to 2D image acquisition devices. (a) Reconstruction based mechanical scanners [2-4]. In this process, 2D image is firstly acquired by mechanical scanners and is then inserted into a 3D image volume using the parameters describing its orientation and position. (b) Reconstruction based freehand scanners [5,6]. Freehand 3D ultrasound is the most commonly used method because of its flexibility. In the reported literature, the $3 \mathrm{D}$ reconstructions based on freehand ultrasound are usually divided into three types: voxel-based method, pixel-based method and the function-based method [7-11]. (c) Reconstruction based 2D array sensor. In

\footnotetext{
${ }^{*}$ Corresponding author: Haijiang Zhu, College of Information Science \& Technology, Beijing University of Chemical Technology, Bei San Huan East Road 15, Chaoyang District, Beijing 100029, China. Tel.: +861064434930; Fax: +861064437805; E-mail: zhuhj@mail.buct.edu.cn.
} 
this approach, 2D phased array sensor is applied to sweep out a volume shaped like a truncated pyramid and to reconstruct the object $[12,13]$.

Currently, B-mode ultrasound images are most commonly used to reconstruct the 3D structure of the examined object [7-11]. A limitation of the 3D ultrasound reconstructions is that the accuracy of these approaches is degraded due to image artifact, distortion and speckle noises in B-mode.

To address these issues, a 3D ultrasound reconstruction algorithm based on ultrasound scanned data is presented. The main motivation of this study is to reconstruct $3 \mathrm{D}$ structure of an object embedded into the TMM samples, thus facilitating medical trainees to make simulation training through the TMM phantom.

This paper is structured as follows: Section 2 presents the 3D reconstruction of the object embedded in the TMM samples; Experimental results and discussion is in Section 3; Section 4 is a conclusion.

\section{Materials and methods}

The proposed method is based on the feature extraction as summarized in Figure 1. The sample is the muscle mimicking materials (see Figure 2) with size $60 \times 50 \times 40 \mathrm{~mm}$, which is made of aqueous gel. This aqueous gel [14] includes polyacrylamide (Tianjin, China), ammonium persulfate (Tianjin, China) and so on. Alumina powder (Shanghai, China) is taken as ultrasound scatter particles. The X-axis and $\mathrm{Y}$ - axis in Figure 2 are the scanning direction, while Z-axis is the acoustic axis direction of the transducer.

Unlike freehand 3D ultrasound reconstruction, the proposed technique does not need to know positions and orientations of the scanners. The reconstruction process in this paper is composed of three steps: One-dimensional echo signals are first smoothed using a two-step varied window filter; 2D images are then generated through aligning one-dimensional smoothed signals; finally, the 3D structure of the object embedded in the TMM sample is modeled using the detected edge points from all $2 \mathrm{D}$ images.

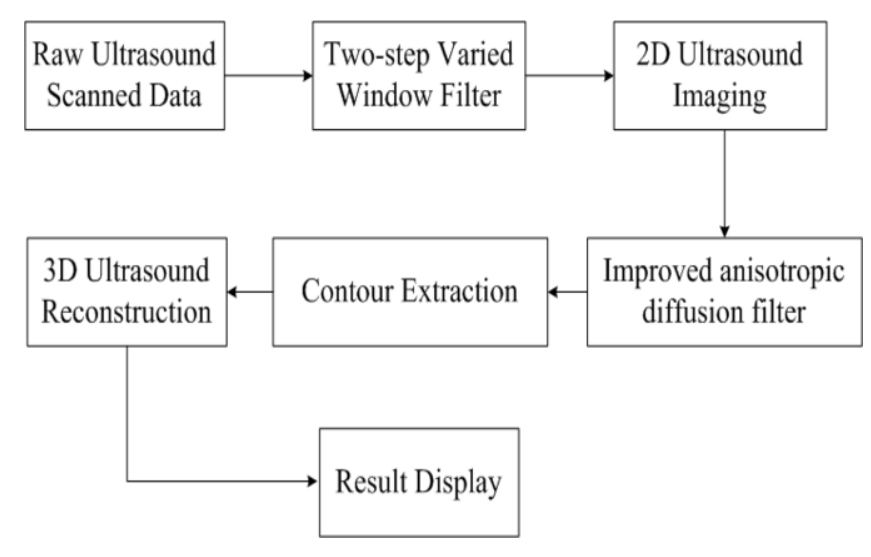

Fig. 1. Summary of the proposed method.

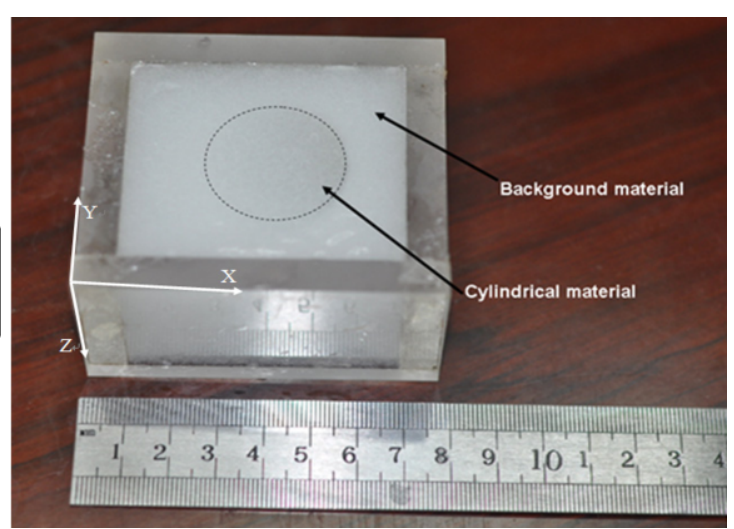

Fig. 2. One of the TMM samples. 


\subsection{Two step varied window filter}

The simple moving average filtering of the observed signals $y(i)$ is assumed as

$$
y(i)=s(i)+n(i)
$$

where $s(i)$ is the original signal, $n(i)$ is Gaussian noise with variance $\sigma_{n}^{2}$ and zero mean. The output $\bar{y}(i)$ of the simple moving average filter can be calculated as

$$
\bar{y}(i)=\sum_{p=-P}^{P} y(i+p) / n_{p}
$$

where $y(i+p)$ is a signal within the window, $P$ is the window size, $n_{p}$ is the number of signals in the windows. In fact, a simple moving average filter may reduce the amplitude of useful signals, which leads to a degraded resolution of the image produced from the backscatter signals. Therefore, a twostep varied windows filter is proposed in this section.

In the first step, the window $W_{1}$ is set to process original backscatter signals. The signal in the window $W_{1}$ is written by $y\left(i+W_{1}\right)$. The local variance $\sigma(i)$ of $y\left(i+W_{1}\right)$ is calculated as

$$
\sigma(i)=\operatorname{var}\left(y\left(i+W_{1}\right)\right)
$$

where $\operatorname{var}(\cdot)$ is the MATLAB function and $\sigma_{n}$ is the total variance of the input signal.

Then an amplitude activity parameter $K(i)$ can be defined as:

$$
K(i)=\frac{\sigma^{2}(i)}{\sigma^{2}(i)+\sigma_{n}^{2}}
$$

In homogeneous regions, $K(i)$ has a small number owing to $\sigma(i)<<\sigma_{n}$. Here, $K(i)$ has a large number in the amplitude points. The max value and min value of the signal in the window $W_{1}$ are respectively estimated from Eqs. (2) and (3).

$$
\begin{aligned}
& y_{\text {max }}=\max \left(y\left(i+W_{1}\right)\right) \\
& y_{\text {min }}=\min \left(y\left(i+W_{1}\right)\right)
\end{aligned}
$$


At last, one signal amplitude threshold $T_{1}$ can be set, and the output signal point in the first step is obtained from Eq. (4).

$$
y_{\text {out }}^{1}(i)= \begin{cases}y_{\min }, & K(i)<T_{1} \\ y_{\max }, & \text { else }\end{cases}
$$

where $y_{\text {out }}^{1}(i)$ stands for the processed signal in the first step.

Moving the window $W_{1}$ in the original backscatter signal, the output signal in the first step can be estimated. Most of the noise can be suppressed, and most of the signals with the larger peak value are preserved in the experiments.

This paper includes a total of $70 \times 89$ scanning signals, most of which are tested using the first step filter as shown in Figure 3. The first row is original backscatter signal, the second row is the result using the simple moving average filter and the third row is the result using the first step filter. It can be seen that the amplitudes of the output signal using the simple moving average filter are decreased by more than $40 \%$. And the first step filter can still preserve the original amplitude.

However, parts of the data points with lower peak value are still in the output signals of the first step. These data points have effects on imaging in the next. Therefore, less than $5 \%$ data points of the max peak value in the first output signals must be further eliminated.

The purpose of the second step is to refine the output signals of the first step using another window $W_{2}$. Let one data point in the output signals of the first step be $A$, the number of the data points with lower peak value before and after point $A$ be $N_{a}$ and $N_{b}$ respectively. $N_{b}$ and $N_{a}$ at each data point in the window $W_{2}$ may be estimated. The threshold value $T_{2}$ in the second step may be set according to Eq. (5).
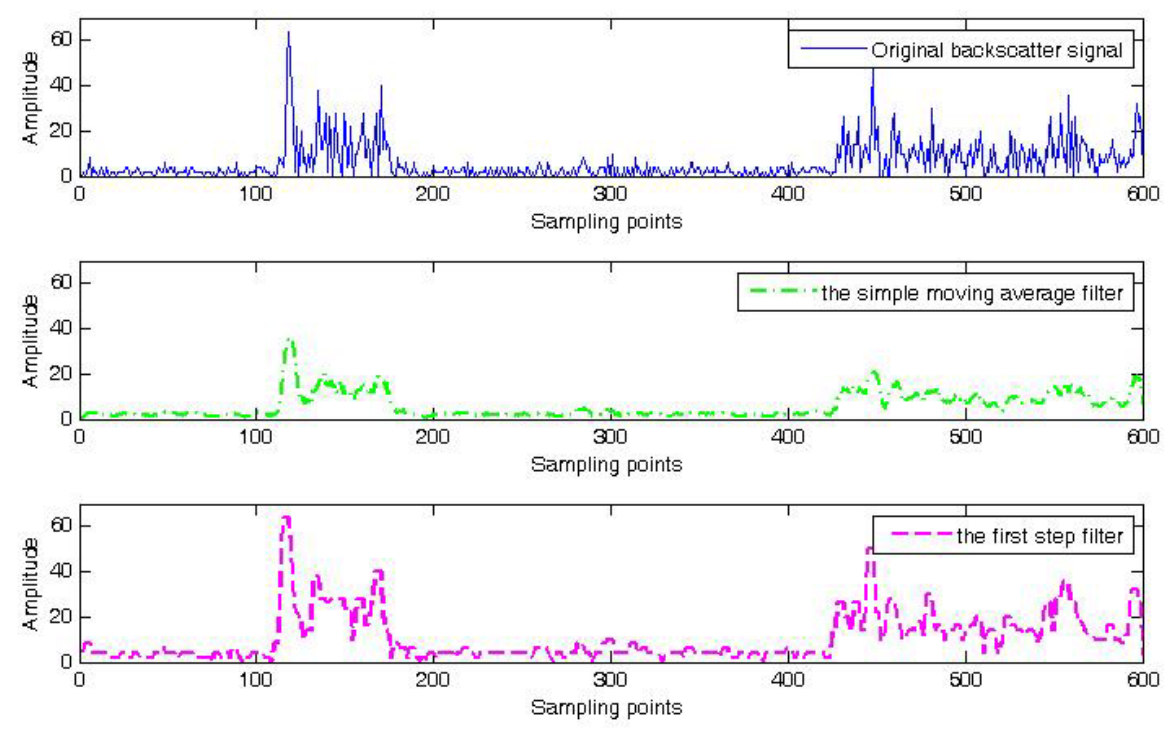

Fig. 3. The processed results using the simple moving average filter and the first step filter. 


$$
y_{\text {out }}^{2}(i)= \begin{cases}B, & N_{a}+N_{b}>T_{2} \\ 0, & \text { other }\end{cases}
$$

where $y_{\text {out }}^{2}(i)$ is the output signal in the second step and $B$ is the setting peak value of the signal. In the experiments, the setting value $B$ can be enlarged to enhance the contrast of the $2 \mathrm{D}$ image.

Ultrasound attenuation images [15] and ultrasound integrated backscatter images [16] were generated by estimating local attenuation coefficients and backscatter coefficients of every pixel in the ultrasonic B scanning image, respectively. The disadvantage of the techniques in $[15,16]$ was its poor resolution. In the proposed method, 1D signal was processed to obtain 2D images. Although it is time consuming, the image has good resolution.

\subsection{Anisotropic diffusion filter with triangular window}

A large number of filters are used to reduce image noise in digital image processing. However, many techniques for removing noise will indiscriminately blur edges. The anisotropic diffusion filter can preserve the edges while removing noise from images [17]. To obtain the edge quickly, the anisotropic diffusion filter is adopted to smooth the 2D scanning images of the TMM sample.

In the Perona [17], an anisotropic diffusion filter is expressed by

$$
\frac{\partial I}{\partial t}=\operatorname{div}\left[c\left(\nabla G_{0} \otimes I \mid\right) \cdot \nabla I\right]
$$

where $I$ is the image intensity, $\operatorname{div}(\cdot)$ is the divergence operator, $c(\cdot)$ is the diffusion coefficient, $\nabla$ denotes the gradient and $G_{0} \otimes I$ is given as a convolution between the image $I$ and a Gaussian kernel $G_{0}$ at the time $t$. In this application, a triangle window function was selected as a kernel function because of the time consumption using the Gaussian kernel in Eq. (6).

The Gaussian kernel, the B-spline kernel were compared with the triangular window kernel in the anisotropic diffusion filter for the 2D image of the TMM sample along the XOZ plane. As shown in Figure 4, the anisotropic diffusion filter with the triangular window kernel can cut down image noise points and detect the slice contour of the cylindrical material in TMM sample.

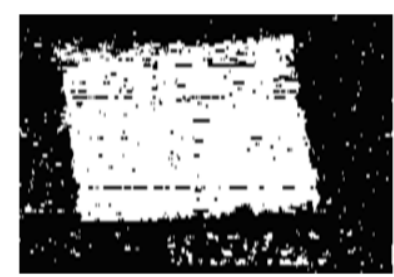

(a)

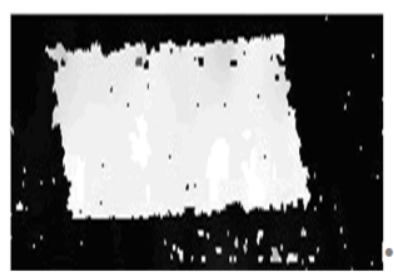

(b)

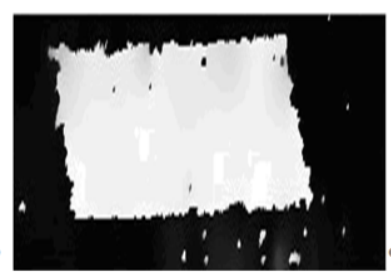

(c)

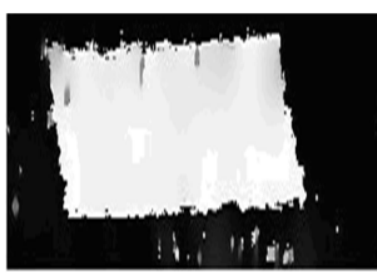

(d)

Fig. 4. The processed results using anisotropic diffusion filter with a different kernel: (a) Original 2D image, (b) Gaussian kernel, (c) B-spline kernel, and (d) Triangular window kernel. 


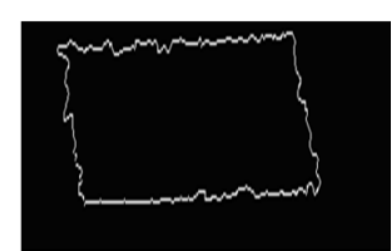

(a)

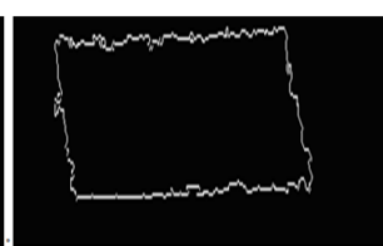

(b)

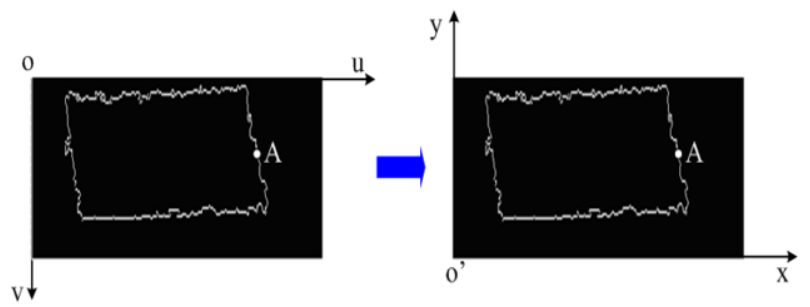

Fig. 5. The extracted edges of (c) and (d) in Figure 4.

Fig. 6. Diagram of edge point coordinate transform.

To acquire the edge points, the edge of the $2 \mathrm{D}$ images smoothed were further extracted. The contour of the region of interest was extracted by using function 'bwperim' in MATLAB. To reduce the influence of uninterested contour, all closed regions in the region of interest were filled up in this step.

The extracted edges of Figures 4(c) and 4(d) are shown in Figures 5(a) and 5(b), respectively. It can be seen that Figure 5(b) can prevent the loss of the edge details from the two edge images.

\subsection{Reconstructed method}

In this section, the 3D image of the TMM sample was constructed using the edge image acquired in Section 2.2.

Firstly, the point cloud of the object contour embedded in the TMM sample was obtained through a serial of its 2D edge images. As shown in Figure 6, the 3D coordinate of the edge point of 2D images is transformed from the pixel coordinates $o$-uv system to the physical coordinates $o^{\prime}$-xy system.

Suppose the length, width and height of TMM sample is $L, W$ and $H$ respectively. The size of 2D image is $U \times V$, and the pixel coordinate of point $A$ on the edge image (see Figure 6) is $[u, v]$. The 3D physical coordinate of edge points in each 2D edge image can be calculated from Eq. (7).

$$
\left\{\begin{array}{c}
x=u \times(L / U) \\
y=(V-v) \times(W / V) \\
z=(N-n) \times(H / M)
\end{array}\right.
$$

where $N$ is the whole number of the $2 \mathrm{D}$ images, $n$ is the number of the image plane apart from the bottom, and $M$ is the number of sampling along the acoustic axis.

The second step is to create a triangle mesh from the point cloud. The crust triangulation $[18,19]$ is used here. However, the crust algorithm is time-consuming due to plenty of calculations while the sampling density is too large. In order to reduce the running time of algorithm, the sampling points are carefully chosen from the estimated 3D cloud data before the crust triangulation.

The algorithm for 3D reconstruction of the object embedded in the TMM sample is outlined as follows.

1. Estimate all 3D points of the object embedded in the TMM sample using Eq. (7) and according to Section 2.1 and Section 2.2.

2. Implement the next algorithm for data reduction.

- Define a given value $\delta$ as the minimum distance in 3D point data. 
- Confirm the maximum point $P_{1}$ along $\mathrm{X}$-axis direction on the same layer.

- Search the nearest point $P_{2}$ in the points that the distance is greater than $\delta$ starting from the point $P_{1}$.

- Regard the point $P_{2}$ as the new reference point and process all points in the same layer.

- Until all layer is searched and processed.

3. Carry out the crust algorithm for the reduced data and output the lighting results.

\section{Experimental results}

\subsection{Ultrasound signal processed results and $2 D$ imaging}

In this section, the two-step varied window filter is tested through different windows and threshold values to process one-dimensional ultrasonic signal.

First, one-dimensional original signal were processed using a simple moving average filter and the first step of the proposed algorithm. The result is shown in Figure 7(a). Black "-" represents the original signals, and cyan "-o" is the results of the simple moving average filter. Red "--" denotes the results using the first step of the proposed algorithm. It can be seen that lots of peak values of the data points are decreased using the simple moving average filter. The proposed algorithm may preserve most data point peak values.

Second, the data points with lower peak value in the output signal of the first step were removed by the second window. The threshold value $T_{2}$ was set as 4 . Figure $7(\mathrm{~b})$ is the results of removing the data points with lower peak value. The output signal amplitude of the second step is set to be 255 in order to enhance image contrast. Black "-", cyan "--" and red "-o" represent the original signal, the output signals of the first step, and the output results of the second step, respectively. These experimental results show that most of the data points with lower peak value in the first output result are eliminated.

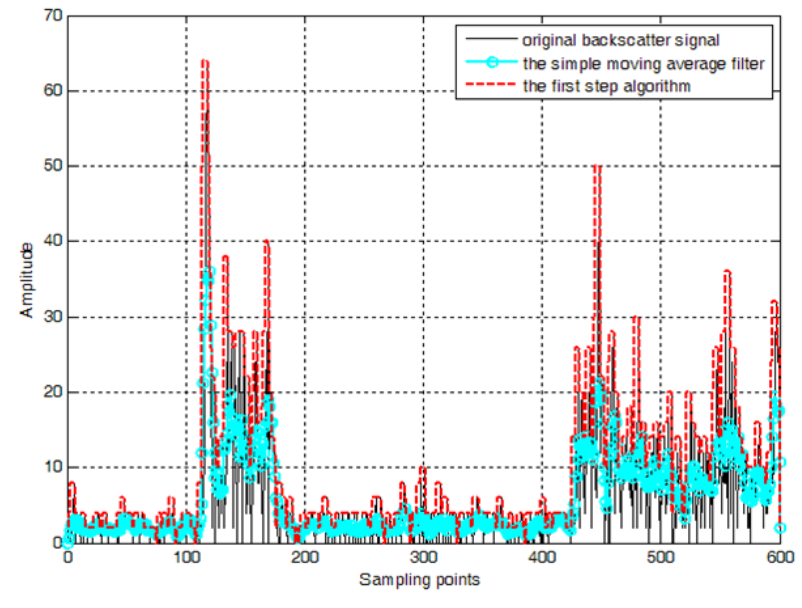

(a)

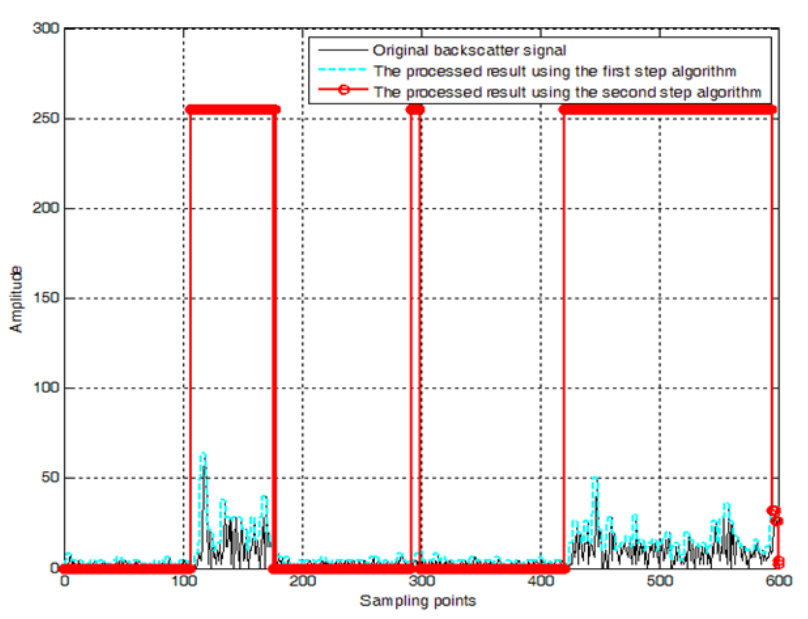

(b)

Fig. 7. The processed results using two-steps varied window filter. 

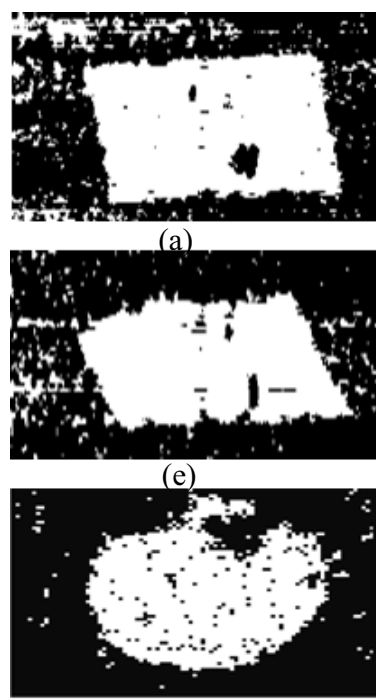

(i)

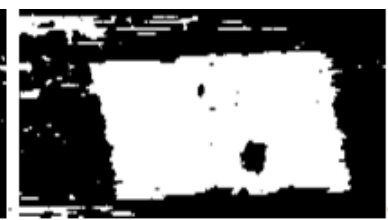

(b)
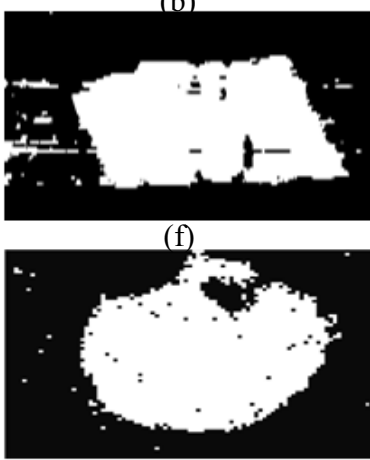

(j)

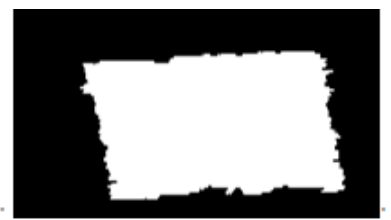

(c)
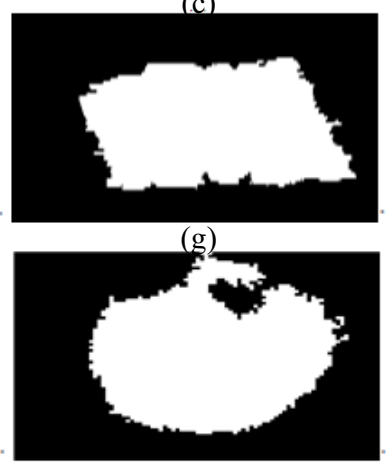

$(\mathrm{k})$

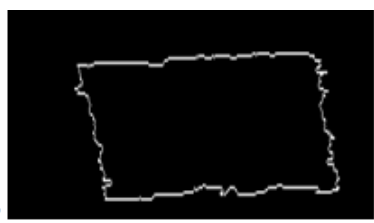

(d)

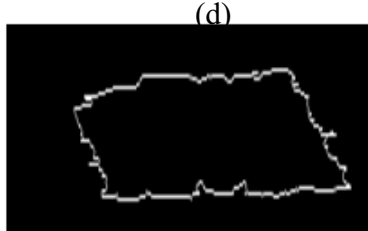

(h)

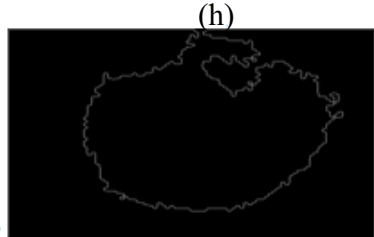

(1)

Fig. 8. The 2D images processing: X-Z plane from (a) to (d), Y-Z plane from (e) to (h) and X-Y plane from (i) to (l).

After the 1D signal processed, the 2D images on the TMM sample along the XOZ plane, YOZ plane and XOY plane directions are obtained through aligning the scanning order of the 1D signal.

The backscattered signal images are shown in Figure 8 for the tissue-mimicking materials sample. Figures $8(\mathrm{a})$ and $8(\mathrm{~b})$ are two $2 \mathrm{D}$ images of the 35th section on the $\mathrm{XOZ}$ plane direction using the twosteps varied window filter. Figures $8(\mathrm{c})$ and $8(\mathrm{~d})$ are the smoothed results using the anisotropic diffusion filter with the triangular window kernel and the extracted edges using the method in Section 2.2, respectively. Similarly, Figures $8(\mathrm{e})-8(\mathrm{~h})$ are the $2 \mathrm{D}$ imaging results of the 30th section on the YOZ plane direction. Figures 8(i)-8(1) are the 2D imaging results of the 340th section on the XOY plane direction. Comparing (a), (e) and (i) with (b), (f) and (j) respectively, one can see that most of the noise points are eliminated after performing the two-step varied window filter. These results prove that the 2D image of TMM sample can be constructed efficiently through the two-step varied window filter. From the edge images in Figures 8(d), 8(h) and 8(1), it can be seen that many edge details are extracted to reconstruct the 3D structure of the TMM sample perfectly.

\subsection{Three dimensional ultrasound imaging results}

To verify the reconstructed algorithm in this paper, a sequence of $2 \mathrm{D}$ images is used to build the $3 \mathrm{D}$ structure of the TMM samples (see Figure 9).

Figure 9 shows the 3D cylinder structures of the object embedded in the TMM sample. Figures 9(a) and 9 (b) are the output of triangulation with $\delta=10$ and the added lighting respectively. Figures 9(c) and 9(d) are the triangulated result of $\delta=40$ and the lighting output, respectively. Intel(R) Core(TM) CPU with $2.93 \mathrm{GHz}$ of memory was used. Running time for the triangulation of the point cloud data is presented in the Table 1. Although its running time is longer than other minimum distance, the result of the cylinder reconstructed using the minimum distance $\delta=10$ appears much smoother. 


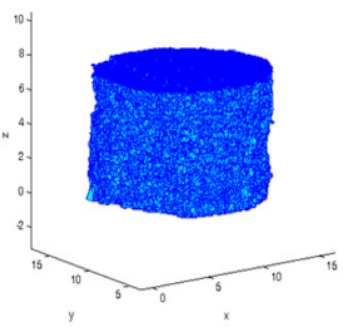

(a)

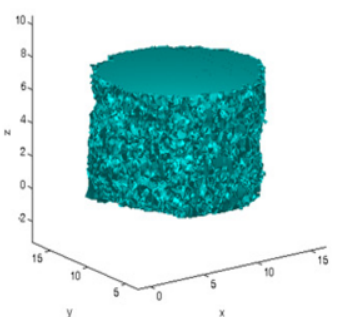

(b)

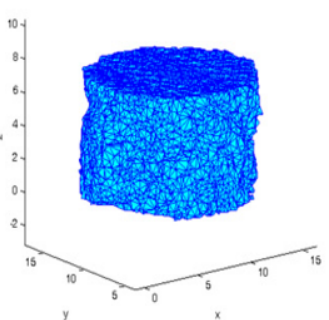

(c)

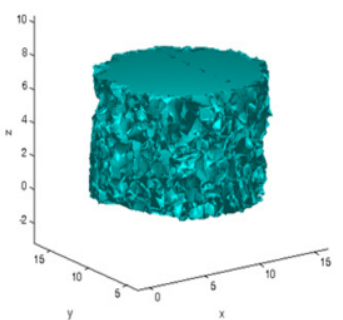

(d)

Fig. 9. The reconstructed 3D cylinder structures of the first TMM sample with a different minimum distance $\delta$ : (a) the triangulated results with $\delta=10$ and (b) the lighting output; (c) the triangulated results with $\delta=40$ and (d) the lighting output.

In addition, the 3D structure of TMM sample from B-mode images was reconstructed using Zhu's technique [20]. Figure 10(a) is one of the B-mode images and Figure 10(b) displays the reconstructed result. It can be seen that there are image artifact and noise in Figure 10(a). Furthermore, B-mode image is blurry, and the object can be only indentified by the expert in ultrasound. As a result, the reconstructed 3D structure of the object from a series of B-mode images is not clear and is hard to identify. This may be due to the B-mode image artifact and noises.

Comparison with Zhu's technique [20] shows that the reconstructed 3D cylinder structure using the proposed method presents a detailed description. And the reconstructed results are less affected by image noise and image artifact as well.

Table1

Running time of triangulation with the different minimum distances

\begin{tabular}{l|l|l|l}
\hline The minimum distance $\delta$ & $\begin{array}{l}\text { Delaunay triangulation time } \\
\text { (second) }\end{array}$ & $\begin{array}{l}\text { Walking time } \\
\text { (second) }\end{array}$ & $\begin{array}{l}\text { Total run time } \\
\text { (second) }\end{array}$ \\
\hline 10 & 1.86 & 2.55 & 4.95 \\
40 & 0.38 & 0.83 & 1.42 \\
\hline 100 & 0.14 & 0.28 & 0.52 \\
\hline
\end{tabular}

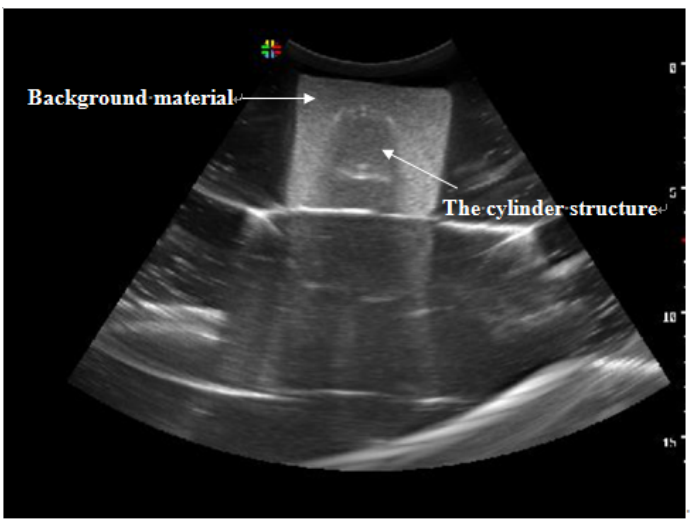

(a)

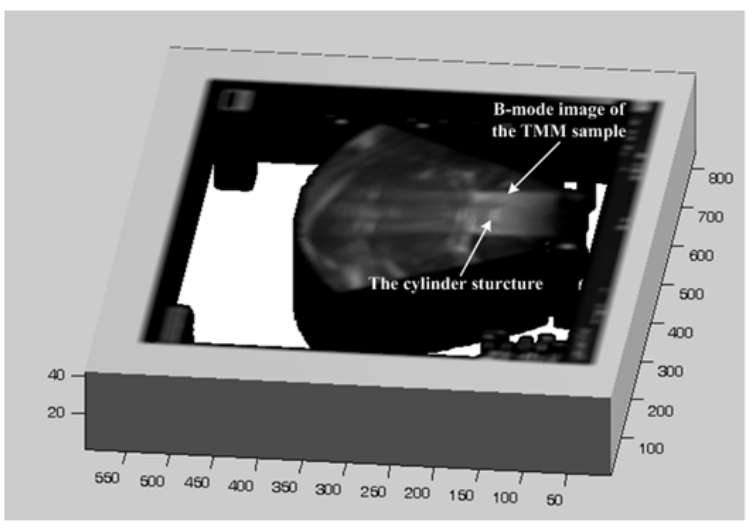

(b)

Fig. 10. The reconstructed 3D structures (b) of the first TMM sample from B-mode images (a). 


\section{Conclusion}

This work has described a 3D structure reconstruction of the TMM sample using the ultrasonic scanning data. The two step varied window filter was proposed to process one-dimensional ultrasonic backscatter signals. And the anisotropic diffusion filter was improved to remove the noise of the 2D images. The 3D point data of the object embedded in the TMM sample were acquired from the extracted edge points of the 2D images. Finally, the 3D model of the object was reconstructed using the crust triangulation for reducing the 3D point data. The experimental results validate the proposed method. Although the proposed method can improve image quality, it is time consuming. The following work will be further carried out to reduce program time under the condition of meeting the reconstruction requirements.

\section{Acknowledgement}

This work was partly supported by the Beijing University of Chemical Technology Interdisciplinary Funds for "Visual Media Computing" and Beijing Training Programme Foundation for the Talents (No. 2012B009016000004). We also thank reviewers' and editors' work for this manuscript.

\section{References}

[1] A. Fenster, D.B. Downey and H.N. Cardinal, Three-dimensional ultrasound imaging, Physics in Medicine and Biology 46 (2001), R67-R99.

[2] A. Papadakis, G. Zacharakis, T. Maris et al., A new optical-CT apparatus for 3-d radiotherapy dosimetry: Is free space scanning feasible? IEEE Transactions on Medical Imaging 29 (2010), 1204-1212.

[3] G. Puy, J.P. Marques, R. Gruetter et al., Spread spectrum magnetic resonance imaging, IEEE Transactions on Medical Imaging 31 (2012), 586-598.

[4] B. Ryu, J. Bax, C. Edirisinge et al., Prostate brachytherapy with oblique needles to treat large glands and overcome pubic arch interference, International Journal of Radiation Oncology Biology Physics 83 (2012), 1463-1472.

[5] H. Yu, M. Pattichis, C. Agurto and G. Beth Goens, A 3D freehand ultrasound system for multi-view reconstructions from sparse 2D scanning planes, Bio-Medical Engineering Online 10 (2011).

[6] A. Lang, P. Mousavi, S. Gill, G. Fichtinger and P. Abolmaesumi, Multi-modal registration of speckle-tracked freehand 3D ultrasound to CT in the lumbar spine, Medical Image Analysis 16 (2012), 675-686.

[7] A. Karamalis, W. Wein, O. Kutter and N. Navab, Fast hybrid freehand ultrasound volume reconstruction, Proceedings of Medical Imaging in SPIE 7261 (2009), 14-18.

[8] G.M. Treece, R.W. Prager, A.H. Gee and L. Berman, Correction of probe pressure artifacts in freehand 3D ultrasound, Medical Image Analysis 6 (2002), 99-214.

[9] W. Huang and Y. Zheng, MMSE reconstruction for 3D freehand ultrasound imaging, International Journal of Biomedical Imaging 1 (2008), 1-8.

[10] Q.H. Huang and Y.P. Zheng, Volume reconstruction of freehand three-dimensional ultrasound using median filters, Ultrasonics 48 (2008), 182-192.

[11] P. Toonkum, N.C. Suwanwela and C. Chinrungrueng, Reconstruction of 3D ultrasound images based on Cyclic Regularized Savitzky-Golay filters, Ultrasonics 51 (2011), 136-147.

[12] O.V. Solberg, F. Lindseth et al., 3D ultrasound reconstruction algorithms from analog and digital data, Ultrasonics 51 (2011), 405-419.

[13] M. Karaman, I.O. Wygant, O. Oralkan and B.T. Khuri-Yakub. Minimally redundant 2D array designs for 3D medical ultrasound imaging, IEEE Transactions on Medical Imaging 28 (2009), 1051-1061.

[14] T. Lin, Q. Jin, H. Yao and H. Zheng, Preparation and characterization of a new ultrasound tissue-mimicking phantom, Bulletin of Advanced Technology Research 4 (2010), 29-33.

[15] E. Walach, A. Shmulewitz, Y. Itzchak et al., Local tissue attenuation images based on pulsed-echo ultrasound scans, IEEE Transactions Biomedical Engineering 36 (1989), 211-221. 
[16] I.J. Thomas, B. Barzilai, J.E. Perez et al., Quantitative real-time imaging of myocardium based on ultrasonic integrated backscatter, IEEE Transactions on Ultrasonics Ferroelectrics and Frequency Control (UFFC) 36 (1989), 466-470.

[17] P. Perona and J. Malik, Scale-space and edge detection using anisotropic diffusion, IEEE Transactions on Pattern Analysis and Machine Intelligence 12 (1990), 629-639.

[18] N. Amenta, M. Bern and M. Kamvysselis, A new Voronoi-based surface reconstruction algorithm, Proceedings of the 25th Annual Conference on Computer Graphics and Interactive Techniques 32 (1998), 415-421.

[19] B. Mederos, N. Amenta, L. Velho and L.H. Figueiredo, Surface reconstruction from noisy point clouds, Eurographics Symposium on Geometry Processing 255 (2005), 53-62.

[20] M. Zhu and S.E. Salcudean, Real-time image-based B-mode ultrasound image simulation of needles using tensorproduct interpolation, IEEE Transactions on Medical Imaging 30 (2011), 1391-1400. 